\title{
Day surgery in the elderly: pain as fifth vital parameter
}

\author{
A Corcione*, M T Di Dato \\ From de Senectute: Age and Health Forum \\ Catanzaro, Italy. 5-7 December 2009
}

\section{Background}

Pain control should be a subject of training in day surgery and specific updating courses for medical and nursing staff in the day hospital and in general for specialized personnel should be done. The ABC acronym may help to remember the fundamental points of pain treatment in day surgery: - assessment: systematic evaluation of pain as the 5 th vital parameter; balanced analgesia: prefer multimodal techniques with the association of nonopioids, opioids and locoregional anesthesia; - continuous audit: continuous verification of patient satisfaction levels; - discharge: discharge the patient with written instruction for home treatment of pain, including details on rescue doses; -education: the education process of the staff (anesthetists, surgeons, nurses) should be continuous and also involve physicians in general medicine.

\section{Materials and methods}

We have evaluated pain management satisfaction in elderly postoperative patients, defining pain management strategies documented in the medical record (MR) that predict patient satisfaction. 56 postsurgical patients aged 65 and older, undergoing elective day surgery procedures (Hernia repair) were surveyed regarding satisfaction with pain management in the first 24 hours postsurgery and the survey results summarized in a score. Pain management variables (patient education, pharmacological and nonpharmacological interventions, demographic variables, and surgery and anesthesia information) were abstracted from their MR. The correlation between the satisfaction score and MR variables was studied.

\section{Discussion}

Sixty-two percent of patients experienced severe postoperative pain, yet $87 \%$ reported being satisfied with the treatment. The mean satisfaction score + standard deviation was $59.3+10.8$ (range 10.6-84.3; potential range $0-100$, higher score $=$ higher satisfaction). MR variables explained $14 \%$ of the satisfaction score variation. The worst pain intensity in the first 24 hours postsurgery as documented in the MR was the most powerful predictor of satisfaction. Other predictors associated with satisfaction were younger age, male sex, preoperative education, shorter recovery room stay, analgesic given through oral route, and NSAIDS (compared with other analgesics).

\section{Conclusions}

Pain in elderly surgical patients remains Undermanaged, in day surgery too. Simple strategies such as emphasizing preoperative education may have a large effect in pain management. This study developed a validated patient satisfaction score and a MR instrument to assist in monitoring pain management quality at home.

Published: 19 May 2010

\section{Reference}

1. Sauaia A, Sung joon Min, Leber C, Erbacher K, Abrams F, Fink R RN: Postoperative Pain Management in Elderly Patients: Correlation Between Adherence to Treatment Guidelines and Patient Satisfaction. JAGS 2005, 53(z):274-282.

doi:10.1186/1471-2318-10-S1-A102

Cite this article as: Corcione and Di Dato: Day surgery in the elderly: pain as fifth vital parameter. BMC Geriatrics 2010 10(Suppl 1):A102. 